
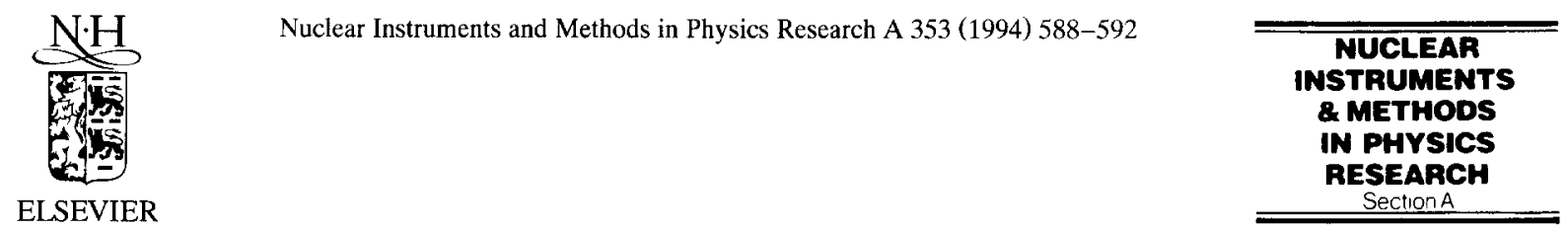

\title{
A position sensitive $\beta-\gamma$ coincidence technique for multiplexed gamma spectrometry of many small samples
}

\author{
Y.K. Dewaraja ${ }^{\text {a,* }}$, Z. He ${ }^{\text {a }}$, R.F. Fleming ${ }^{\text {a }}$, D.K. Wehe ${ }^{\text {a }, ~ S . V . ~ G u r u ~}{ }^{\text {a }}$, J.C. Ferreira ${ }^{\text {a }}$, \\ R.H. Fleming ${ }^{b}$ \\ ${ }^{a}$ Department of Nuclear Engineering, Phoenix Memortal Laboratory, Unwersity of Michigan, Ann Arbor, MI 48109-2100, USA \\ ${ }^{\mathrm{b}}$ Charles Evans \& Associates, 301, Chesapeake Drive, Redwood City, CA 94063, USA
}

\begin{abstract}
A technique based on $\beta-\gamma$ coincidence has been developed to perform multiplexed gamma-ray spectrometry of small samples using a single gamma-ray detector and a position sensitive beta detector. A system is described that uses a position sensitive photomultiplier tube coupled to a thin plastic scintillator as the beta imaging detector. Multiplexed gamma-ray spectrometry is demonstrated by results obtained with this system for a $4 \times 4$ array of $\mathrm{Au}$, $\mathrm{Co}$ and $\mathrm{Ag}$ samples. The advantages of this technique over gamma spectrometry on individual samples are the substantial reduction in total counting time and the reduction in background, which are especially significant in neutron activation analysis of particles.
\end{abstract}

\section{Introduction}

The concept of multiplexed gamma-ray spectrometry is based on the previously reported Imaging Neutron Activation Analysis (Imaging NAA) technique [1,2]. Multiplexed gamma spectrometry is applicable to most elements that undergo charged particle emission from $\alpha$ or $\beta$ decay, followed by the coincident emission of a gamma-ray. An array of samples is analyzed using a detection system consisting of a single gamma-ray detector, a 2-D charged particle imaging detector and a coincidence circuit. When a charged particle and a gamma-ray are detected in coincidence, the charged particle position signal determines the location of the original disintegration, i.e., the sample from which the gamma-ray originated is localized. The technique is essentially limited to samples whose thicknesses are small compared to the charge particle range in the sample.

The majority of the characteristic gamma rays used in conventional neutron activation analysis (NAA) are emitted in coincidence with a charged particle, which is often a beta particle. The only exceptions are the gamma rays due to some isomeric transitions such as the $439 \mathrm{keV}$ gamma ray of ${ }^{69} \mathrm{Zn}$. Radionuclides that decay purely by electron capture (EC) such as ${ }^{51} \mathrm{Cr}$ can also be analyzed by this technique because the low energy Auger electrons can be detected by some imaging systems [3]. Thus, multiplexed gamma spectrometry is highly suitable in neutron activa-

\footnotetext{
${ }^{*}$ Corresponding author.
}

tion analysis of small samples. Submicrogram cosmic dust particles have been analyzed by conventional NAA using long counting times and large $\mathrm{Ge}$ detectors in a low background facility [4]. Multiplexed spectrometry of such particles will substantially reduce the total counting time compared with counting the particles individually. Also, since the gamma spectrum that is measured is a coincidence spectrum, the low background requirement can be relaxed.

The feasibility of the technique is demonstrated by results obtained using a position sensitive photomultiplier tube (PSPMT) as the charged particle imaging detector. Multiplexed gamma spectrometry using the previously reported Imaging NAA instrument is also discussed.

\section{The PSPMT / Ge detector system}

A position sensitive photomultiplier tube coupled to a 1 $\mathrm{mm}$ thick plastic scintillator (NE102A) is used for charged particle imaging. The commercially available PSPMT (Hamamatsu R2487) consists of a conventional bialkaline photocathode, a 12 stage mesh dynode structure and two planes of crossed wire anodes of $X$ and $Y$, respectively [5]. The secondary electron charge cloud is collected by each anode plane and divided by the external resistor chain. Two dimensional position information can be obtained from the centroid calculation: $X=\left(X_{1}-X_{2}\right) /\left(X_{1}+X_{2}\right)$ and $Y=\left(Y_{1}-Y_{2}\right) /\left(Y_{1}+Y_{2}\right)$, where $X_{1}, X_{2}, Y_{1}, Y_{2}$ are the outputs from the charge sensitive preamplifiers at the ends of the two resistor chains. The energy of the original 
charged particle interaction can be obtained from the sum signal $E_{\beta}=\mathrm{X}_{1}+\mathrm{X}_{2}+\mathrm{Y}_{1}+\mathrm{Y}_{2}$.

The samples are placed directly on the scintillator and positioned between the PSPMT and the $\mathrm{Ge}(\mathrm{Li})$ detector as shown in Fig. 1. The $\mathrm{Ge}(\mathrm{Li})$ crystal diameter is $4.4 \mathrm{~cm}$ while the PSPMT active area is $6 \times 5.5 \mathrm{~cm}^{2}$. As shown in the figure, the two inputs to the coincidence unit (Ortec 414) are the PSPMT beta sum signal and the $\mathrm{Ge}(\mathrm{Li})$ gamma-ray signal. The PSPMT signal arrives before the $\mathrm{Ge}(\mathrm{Li})$ signal, hence it was delayed by about $6 \mu \mathrm{s}$. The coincidence resolving time was set to its maximum value of $110 \mathrm{~ns}$. Each of the four PSPMT beta position signals and the $\mathrm{Ge}(\mathrm{Li})$ gamma ray energy signal are sent to an input channel of the data acquisition board via a peak detection circuit. The computer data acquisition board (Analog Devices RTI-860) has 16 input channels and a 12-bit resolution analog to digital converter. When a coincidence trigger signal occurs $X_{1}, X_{2}, Y_{1}, Y_{2}$ and $E_{\gamma}$ are digitized and saved in a list file. The centroid calculation to determine $X-Y$ positions is performed by software after the data acquisition. The gamma spectrum of an individual sample is obtained by applying an $X-Y$ window to include only the image area of that sample and sorting through the coincidence list file for the corresponding $E_{\gamma}$.

\section{Results using the PSPMT / Ge detector system}

To demonstrate multiplexed gamma spectrometry, well defined test samples were prepared by evaporating pure metals onto a silicon substrate through a photoresist mask. Gold and silver were deposited directly on silicon while cobalt was deposited onto a silicon wafer coated with a thin layer of chromium. The samples were $0.8 \mathrm{~mm}$ in diameter and the metal thickness ranged from 40 to 100 $\mathrm{nm}$. The Au samples were irradiated in a neutron flux of $4 \times 10^{12} / \mathrm{cm}^{2} \mathrm{~s}$ for a period of $10 \mathrm{~min}$ while the $\mathrm{Ag}$ and

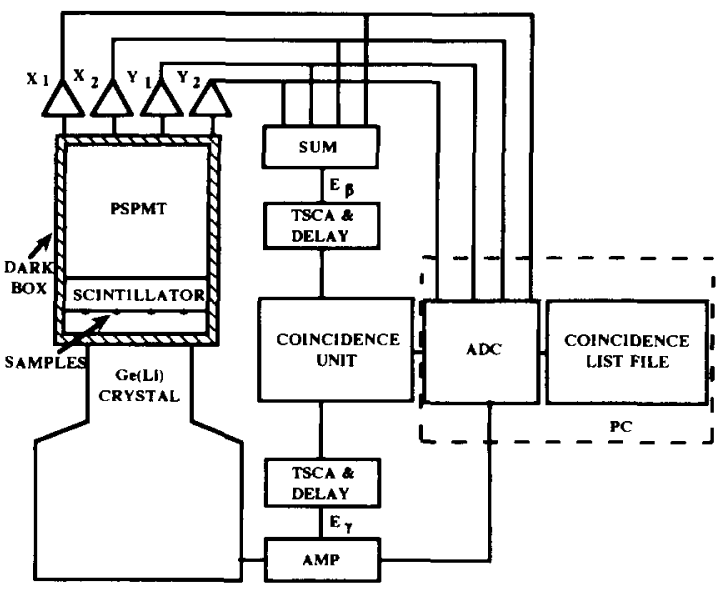

Fig. 1. Block diagram of the PSPMT/Ge detector system.
Co samples were irradiated for a period of few days at the same flux. Fourteen of the Au samples and one each of the $\mathrm{Co}$ and $\mathrm{Ag}$ samples were arranged in a $4 \times 4$ array with 1 $\mathrm{cm}$ spacing. They were covered with a thin paper $(\sim 20$ $\mu \mathrm{m})$ to avoid contamination and placed directly on a $1 \mathrm{~mm}$ plastic scintillator coupled to the PSPMT. The coincidence beta image of this array is shown in Fig. 2a. The gamma-ray spectra of disintegrations originating within the three rectangular areas indicated in the image are shown in Figs. $2 \mathrm{~b}-2 \mathrm{~d}$. The three spectra clearly correspond to $\mathrm{Au}, \mathrm{Ag}$ and Co samples and show little or no interference from either surrounding samples or chance coincidences. The Au sample spectrum does not show any $\mathrm{Ag}$ or Co interference, while the $\mathrm{Au}$ interference in both the $\mathrm{Ag}$ and Co sample spectra amounts to less than $0.5 \%$ of the total Au.

Multielement particles were also analyzed by multiplexed gamma spectrometry. The material used was silicate glass that had been specially prepared for use as standards in neutron activation analysis of cosmic dust particles [4]. Four particles (approximately $200 \mu \mathrm{m}$ in size) were multiplexed and the individual gamma spectra were resolved. The ${ }^{47} \mathrm{Sc},{ }^{59} \mathrm{Fe},{ }^{24} \mathrm{Na}$, and ${ }^{198} \mathrm{Au}$ gamma peaks were observed.

\section{Coincidence efficiency, spatial resolution, and accu- racy}

The coincidence efficiency corresponding to each photopeak is determined from the ratio of the photopeak area of the coincidence gamnia spectrum to the photopeak area of the total gamma spectrum. This efficiency is equivalent to the beta detection efficiency of the imaging detector [2]. Coincidence efficiencies of $34 \%$ and $16 \%$ were measured for ${ }^{198} \mathrm{Au}$ and ${ }^{110 \mathrm{~m}} \mathrm{Ag}$, respectively. Based on geometry alone, the best achievable coincidence efficiency of the PSPMT/Ge system is $50 \%$ for thin samples where self attenuation of the beta particles can be ignored. However, the measured coincidence efficiency can be substantially lower depending on the energy of the beta particles and the low level noise threshold. The beta sum (energy) signal, $E_{\beta}$, determines if an event is above or below the threshold. Since the beta energy spectrum extends down to zero energy, the noise threshold will eliminate true events as well. This loss in efficiency is greatest for beta emitters with low average energy which is demonstrated by the gamma intensities in the $\mathrm{Ag}$ coincidence spectrum (Fig. $2 \mathrm{c}$ ). Table 1 gives the gamma-ray intensities and average beta energies associated with the decay of ${ }^{110 \mathrm{~m}} \mathrm{Ag},{ }^{60} \mathrm{Co}$ and ${ }^{198} \mathrm{Au}$. Although the $885 \mathrm{keV}$ gamma ray of ${ }^{110 \mathrm{~m}} \mathrm{Ag}$ has a much higher intensity than the $937 \mathrm{keV}$ gamma, these two photopeaks have almost the same intensity in the coincidence spectrum because of their different beta energies. The $764 \mathrm{keV}$ and $1384 \mathrm{keV}$ gamma rays are not observed in the coincidence spectrum because the beta particles (average energy of $22 \mathrm{keV}$ ) are eliminated by the 
lower level threshold of the PSPMT or are attenuated in the thin paper that covers the samples. Note also that the $320 \mathrm{keV}$ photopeak of ${ }^{51} \mathrm{Cr}$ is not observed in Fig. 2d, although the Co was deposited onto a $\mathrm{Cr}$ layer. Therefore, the PSPMT based system is not suitable for analysis of radionuclides that decay by $\mathrm{EC}$ or have a very low beta endpoint energy.

A spatial resolution of $2.5 \mathrm{~mm}$ (FWHM) was measured for ${ }^{198} \mathrm{Au}$. The spatial resolution of the image depends both on the characteristics of the scintillator and the energy deposited by the beta particles. The optimum scintillator should provide the maximum number of light photons while minimizing the light spread on the photocathode.
The material should also have a high beta stopping power. $\mathrm{NaI}$ has many of the desired characteristics but the crystal has to be encapsulated because of its hygroscopic nature, making it unsuitable for imaging most beta particles. Although its light output is only $30 \%$ of $\mathrm{NaI}$, a plastic scintillator was used in the present work because it is economical and readily available in any required geometry. As Table 1 indicates, $1 \mathrm{~mm}$ of the plastic is sufficiently thick to stop the majority of beta particles that were analyzed. In order to fully stop the betas from the high end of the ${ }^{198} \mathrm{Au}$ spectrum and to stop higher energy beta emitters such as ${ }^{24} \mathrm{Na}$, a thicker plastic scintillator has to be used. Due to the uncertainly associated with locating
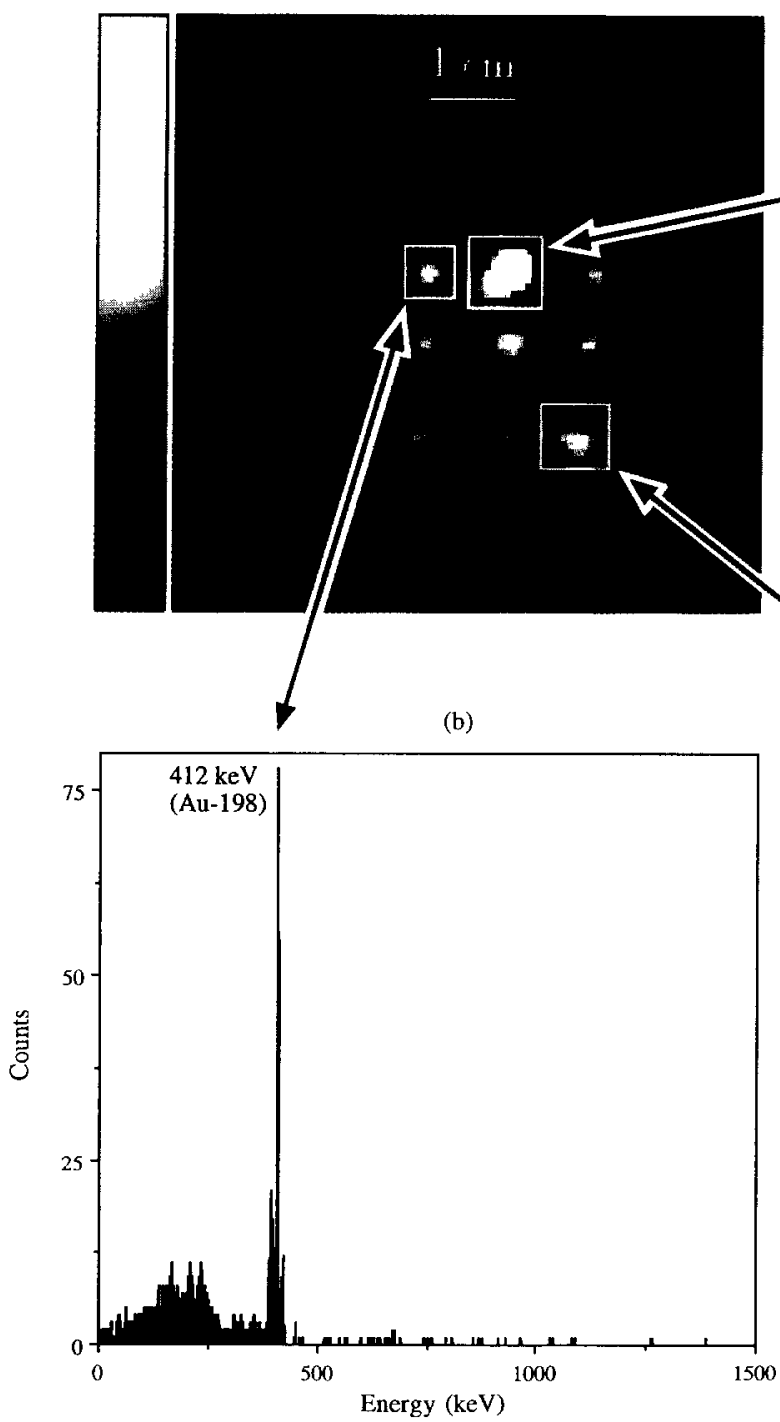

(c)

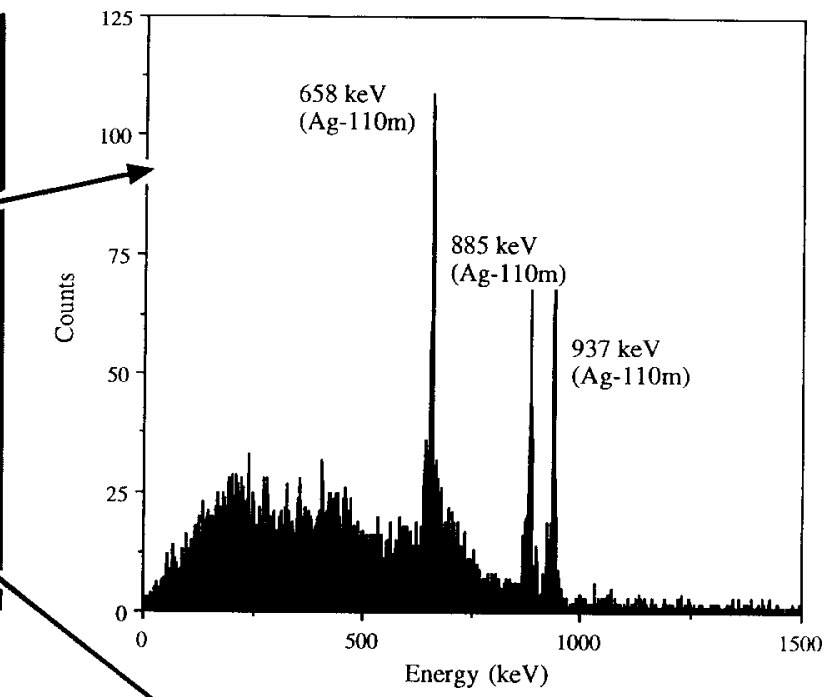

(d)

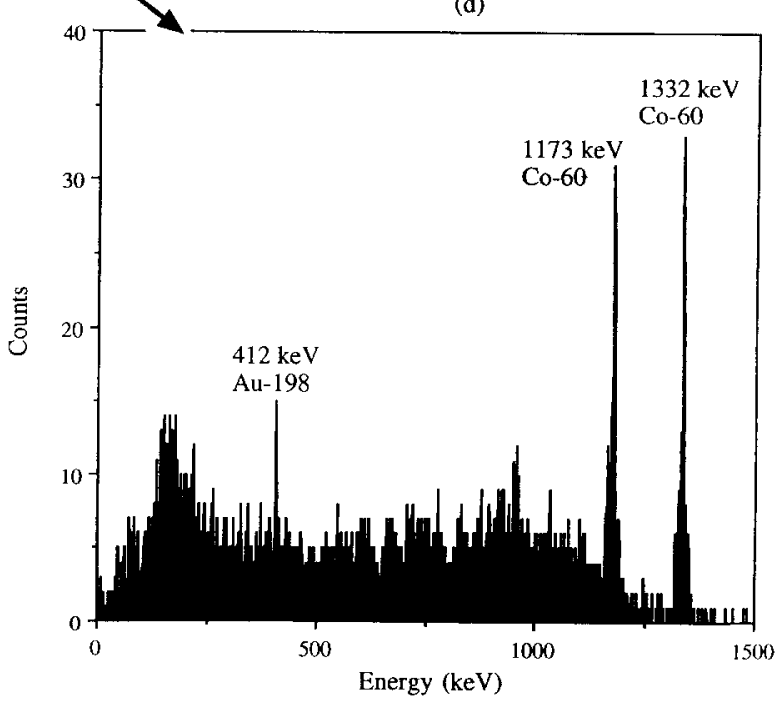

Fig. 2. (a) Coincidence image of an array of neutron activated $\mathrm{Au}, \mathrm{Ag}$ and $\mathrm{Co}$ samples obtained from the PSPMT/Ge system. The separation between samples is $1 \mathrm{~cm}$. (b-d) The local area coincidence gamma spectrum corresponding to the three rectangular regions indicated in (a). 
Table 1

Gamma-ray energies, intensities and average beta energies of ${ }^{110} \mathrm{Ag},{ }^{60} \mathrm{Co}$ and ${ }^{198} \mathrm{Au}$. The range of average energy beta particles in the plastic scintillator [6] is given in the last column

\begin{tabular}{llll}
\hline & $\begin{array}{l}\gamma \text {-ray energy (keV) } \\
\text { and intensity }(\%)\end{array}$ & $\begin{array}{l}\text { Average } \\
\beta \text {-energy } \\
\text { (keV) }\end{array}$ & $\begin{array}{l}\beta \text { range in } \\
\text { plastic }(\mathrm{mm})\end{array}$ \\
\hline${ }^{110 \mathrm{~m}} \mathrm{Ag}$ & $658(94)$ & 69 & 0.08 \\
& $764(22)$ & 22 & 0.01 \\
& $885(73)$ & 83 & 0.09 \\
& $937(34)$ & 149 & 0.20 \\
& $1384(26)$ & 22 & 0.01 \\
${ }^{60} \mathrm{Co}$ & $1173(100)$ & 96 & 0.15 \\
& $1332(100)$ & 96 & 0.15 \\
${ }^{198} \mathrm{Au}$ & $412(96)$ & 315 & 1 \\
\hline
\end{tabular}

the centroid, the spatial resolution of the PSPMT varies inversely in proportion to the square root of the number of light photons [7], which in turn is proportional to the energy deposited by the charged particle. Hence, the spatial resolution should improve when events corresponding to the low end of the beta spectrum are eliminated as demonstrated by the results in Fig. 3. This figure shows the beta intensity profile across four ${ }^{198} \mathrm{Au}$ sources $(1 \mathrm{~cm}$ apart) for two different values of the PSPMT lower level threshold. The improvement in spatial resolution comes at the expense of coincidence efficiency as discussed previously. The higher threshold resulted in a $57 \%$ loss in efficiency in the case of Fig. 3.

The accuracy of multiplexed gamma spectrometry using the PSPMT/Ge system depends on some factors that are not encountered in conventional gamma spectrometry. Interference due to activities of the other samples in the array, radial variation in $\mathrm{Ge}(\mathrm{Li})$ detection efficiency, and

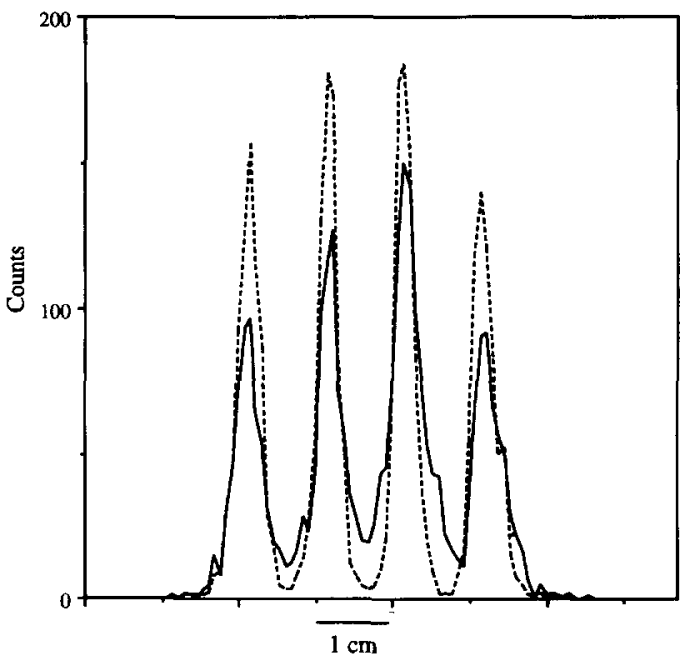

Fig. 3. Beta intensity profile ( $X$ direction) measured for four ${ }^{198} \mathrm{Au}$ sources spaced $1 \mathrm{~cm}$ apart and positioned on the $1 \mathrm{~mm}$ plastic scintillator coupled to the PSPMT. The dashed curve was measured with a higher value of the low level threshold compared with the solid curve.

gain variation within the PSPMT active area need to be considered. The coincidence efficiency is a function of beta energy, the PSPMT noise threshold, and also sample thickness when beta self attenuation becomes significant. Interference from surrounding samples can be minimized by using adequate separation between samples and careful determination of the image area corresponding to each sample. The problems of variation of $\mathrm{Ge}(\mathrm{Li})$ efficiency, tube gain and coincidence efficiency can be overcome if standards of the same geometry are counted at the same positions.

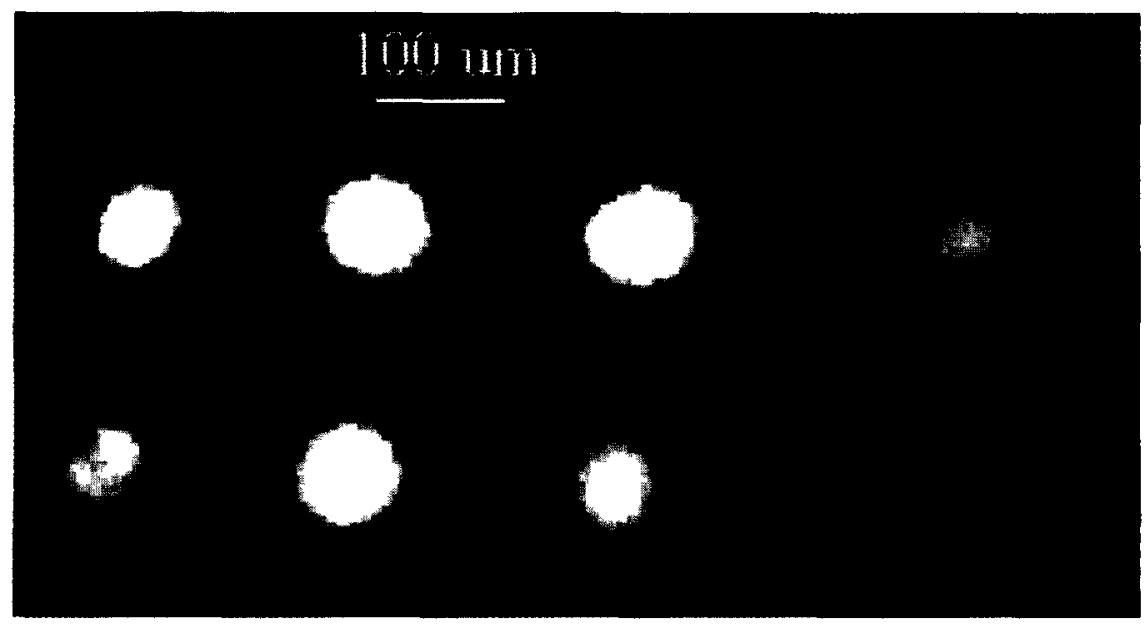

Fig. 4. Image of a test pattern of neutron activated Au dots obtained from the prototype Imaging NAA instrument. The separation between the dots is $200 \mu \mathrm{m}$ center to center. 


\section{Results using the Imaging NAA instrument}

Multiplexed gamma spectrometry was also performed using the prototype Imaging NAA instrument. The same $\mathrm{Ge}(\mathrm{Li})$ detector was used together with the secondary electron based imaging system that has been described in previous literature [1,2]. A test pattern was prepared by evaporating an array of pure Au dots, $100 \mu \mathrm{m}$ in diameter and $200 \mu \mathrm{m}$ center to center. The image due to the ${ }^{198} \mathrm{Au}$ activity is shown in Fig. 4. The gamma spectrum corresponding to each dot was resolved. The high resolution capability of this system allows samples to be positioned very close to one another without significant interferences and negligible variation in $\mathrm{Ge}(\mathrm{Li})$ efficiency at each sample position. Another advantage of this system is its ability to image very low energy beta particles and Auger electrons. The measured coincidence efficiency of this system is low ( $2 \%$ for ${ }^{198} \mathrm{Au}$ ) and the active imaging area is $1 \mathrm{~mm}$ in diameter.

\section{Conclusions}

Multiplexed gamma spectrometry is especially suitable for neutron activation analysis of microscopic particles. Major advantages over conventional gamma spectrometry are the reduction in total counting time and the low background, while the biggest disadvantage is potential interference from other samples in the array. The large active area, and the excellent beta detection efficiency of the PSPMT makes it a highly suitable imaging detector for this technique. The position resolution that was achieved
(2.5 mm FWHM for ${ }^{198} \mathrm{Au}$ ) can be improved by optimal selection of the scintillator material and geometry. A spatial resolution of $0.5 \mathrm{~mm}$ has been reported in pure beta imaging of ${ }^{32} \mathrm{P}$ using a PSPMT coupled to a thin NaI scintillator [8]. The spatial resolution can also be improved by eliminating the beta particles at the low end of the spectrum, but this comes at the expense of efficiency.

\section{Acknowledgements}

The authors are grateful to Dr. Terry Hanson (NE Technology) for providing the plastic scintillators and to Dr. R. Lindstrom (NIST) and Dr. D. Lindstrom (NASA) for providing the glass standards.

\section{References}

[1] R.H. Fleming, R.F. Fleming and Y.K. Dewaraja, submitted to Anal. Chem.

[2] Y.K. Dewaraja, R.F. Fleming, M.A. Ludington and R.H. Fleming, IEEE Trans. Nucl. Sci. NS-41 (4) (1990).

[3] Y.K. Dewaraja, R.F. Fleming and R.H. Fleming, Trans. Am. Nucl. Soc., 68 San Diego, Ca, USA, 1993. p. 4.

[4] D.J. Lindstrom, Nucl. Instr. and Meth. A 299 (1990) 584

[5] Specification sheet for R-2487 PSPMT, Hamamatsu Photonics K.K. Electron Tube Division, Japan.

[6] Technical data NE102A plastic scintillator, NE Technology Limited, Scotland.

[7] H. Kume, S. Muramatsu and M. Iida, IEEE Trans. Nucl. Sci. NS-33 (1) (1986) 359.

[8] W.J. McGann, L. McConchie and G. Entine, Nucl. Instr. and Meth. A 299 (1990) 511. 